\title{
Modelos matemáticos y la calidad del servicio aplicados al transporte urbano en Riobamba.
}

Mathematical models and quality of service applied to urban transport in Riobamba.

Monserrath Amparo Padilla Muñoz. ${ }^{1}$, José Luis Llamuca Llamuca. ${ }^{2}$, Pablo Ricardo Calderón Limaico. ${ }^{3}$ \& Jenny Margoth Villamarín Padilla. ${ }^{4}$

\section{Recibido: 10-03-2019 / Revisado: 15-03-209 /Aceptado: 04-04-2019/ Publicado: 13-05-2019}

\begin{abstract}
.
DOI: https://doi.org/10.33262/cienciadigital.v3i2.2.466

The city of Riobamba is located relatively in the center of Ecuador, it is a city that is known as the city of the first fruits, for the activities of national relevance that occurred at the time, for its contribution to growth and development in different sectors, transport being a very important sector in the middle.

Most of the inter-provincial and interprovincial Inter public road transport Cooperatives must pass through the city of Riobamba to reach the different cities of the coastal, mountain and eastern regions, due to their geographical location.

This type of transport mobilizes a large number of people and transfers a good part of the merchandise that will be used for the production and commercialization of goods nationwide.

A historic and emblematic city for the country, should not have problems in the issue of urban land transport internally and in the roads that connect with the surrounding cities, the quality of service provided by those involved in the transport sector, not only that allow to improve the income of the service providers, also allow to improve the income of other sectors that are related to transport.
\end{abstract}

\footnotetext{
${ }^{1}$ Escuela Superior Politécnica del Chimborazo, Facultad de Administración de Empresas, Riobamba Ecuador, momserrath_1104@yahoo.es

${ }^{2}$ Escuela Superior Politécnica del Chimborazo, Facultad de Administración de Empresas, Riobamba Ecuador, pepes.j1@gmail.com

${ }^{3}$ Escuela Superior Politécnica del Chimborazo, Facultad de Administración de Empresas, Riobamba Ecuador, pablo.calderon@espoch.edu.ec

${ }^{4}$ Escuela Superior Politécnica del Chimborazo, Facultad de Administración de Empresas, Riobamba Ecuador, jenny.villamarin@espoch.edu.ec
} 
Keywords: Transport, Mathematics, Social Responsibility, Quality, Urban Transport

\section{Resumen.}

La ciudad de Riobamba está ubicada relativamente en el centro del Ecuador, es una ciudad que se la conoce como la ciudad de las primicias, por las actividades de relevancia nacional que se dieron en su momento, por su aportación al crecimiento y desarrollo en diferentes sectores, siendo el transporte un sector muy importante en el medio.

La mayor parte de las Cooperativas de transporte público terrestre Inter cantonal e interprovincial, deben pasar por la ciudad de Riobamba para llegar a las diferentes ciudades de las regiones de la costa, sierra y oriente, debido a su ubicación geográfica.

Este tipo de transporte moviliza a un gran número de personas y traslada una buena parte de las mercaderías que serán utilizadas para la producción y comercialización de bienes a nivel nacional.

Una ciudad histórica y emblemática a para el país, no debería tener problemas en el tema del transporte terrestre urbano a nivel interno y en las vías que comunican con las ciudades aledañas, la calidad de servicio que brinden los involucrados en el sector transporte, no solamente que permiten mejorar los ingresos de los prestadores de servició, permiten también mejorar los ingresos de otros sectores que tienen relación con el transporte.

La ciudad de Riobamba en los últimos años ha crecido considerablemente tanto vertical y horizontal, las líneas de transporte público no tienen una planificación moderna en cuanto a este problema de crecimiento causando problemas para la ciudadanía, a esto sumamos también la falta reordenamiento territorial.

Palabras claves: Transporte, Matemáticas, Responsabilidad Social, Calidad, Transporte Urbano.

\section{Introducción.}

Esta investigación busca determinar correctivos a adecuados para sugerir una planificación vehicular en nuestra ciudad pues desde hace algún tiempo encontramos ciertas irregularidades concerniente en la planificación del servicio que presta el transporte urbano en la ciudad de Riobamba.

Este estudio será de gran beneficio porque se puede evidenciar lo que sucede, determinar las posibles mejoras a aplicar en el área de servicio, pues como se encontró calidad no existe en el servicio 
El tributo que se dará es que cuente con los métodos definidos para poder realizar sus nuevas actividades de la manera correcta como lo dicta la ley,

Según el plan Mundial para el Decenio de Acción para la seguridad Vial 2011 - 2020, cerca de 1,3 millones de personas fallecen por motivos de accidentes de tránsito, 3.000 defunciones al día, lo cual es un grave problema para la humanidad en general y de manera directa para aquellos familiares que pierden a sus seres queridos y para ellos que pierden al sostén de familia en accidentes de tránsito de los cuales en algunos casos no son los responsables directos.

La Asociación Latino - Americana de Sistemas Integrados (SIBRT), conformado por representantes de agencias de Transporte Urbano de las principales ciudades de los siguientes países: Brasil, Chile, Colombia, Ecuador y México; contribuyen a mejorar el transporte masivo en América Latina, buscando la cooperación en el ámbito profesional, tecnológico, científico,

financiero, comercial e institucional, para fortalecer la capacidad y la efectividad de los distintos organismos interesados y la calidad de sus sistemas.

La Movilidad Sustentable de América Latina (MUSAL), que es una entidad cuya visión es constituir la coordinación de los gobiernos de las ciudades latinoamericanas para concretar el compromiso de acción conjunta y permanente, para alcanzar una movilidad urbana de alta calidad para todos.

Los agraciados directos de esta investigación es la misma entidad que brindara sus servicios de una manera eficiente y eficaz para los usuarios, los agraciados indirectos serían las otras entidades de otros cantones que tenga la planificación, regulación y control dentro de su competencia, teniendo una guía para asumir competencias de modelo de gestión de categoría A,

\section{Antecedentes Investigativos}

Para poder realizar este trabajo de investigación se recabo información de manera local para poder indagar en el ámbito de las competencias de transporte y la planificación dentro de la ciudad de Riobamba

La Información recabada en una primera etapa "análisis matemático en la calidad en el servicio urbano en la ciudad de Riobamba para futuros proyectos de inversión publica" nos sirvió para emitir la segunda conclusión en cuanto a la calidad de este servicio de transporte urbano, 
La información obtenida en la encuesta de las siguientes 7 preguntas detallada que a continuación muestra la insatisfacción de parte de los usuarios las mismas que para nuestro análisis fue tomado con las quejas resultantes de la ciudadanía de la ciudad

Tabla1. Rangos de Edad de los Encuestados

\begin{tabular}{|l|r|r|}
\hline \multicolumn{1}{|c|}{ RANGOS } & RESULTADOS & PORCENTAJE \\
\hline De 19 Hasta 25 & 150 & $37,97 \%$ \\
\hline De 26 Hasta 32 & 67 & $16,96 \%$ \\
\hline De 12 Hasta 18 & 37 & $9,37 \%$ \\
\hline De 33 Hasta 39 & 37 & $9,37 \%$ \\
\hline De 40 Hasta 46 & 35 & $8,86 \%$ \\
\hline De 47 Hasta 53 & 29 & $7,34 \%$ \\
\hline De 54 Hasta 60 & 15 & $3,80 \%$ \\
\hline De 68 Hasta 74 & 11 & $2,78 \%$ \\
\hline De 61 Hasta 67 & 10 & $2,53 \%$ \\
\hline De 75 Hasta 81 & 4 & $1,01 \%$ \\
\hline Total general & $\mathbf{3 9 5}$ & $\mathbf{1 0 0 , 0 0 \%}$ \\
\hline
\end{tabular}

Fuente: Investigación de campo, Equipo de Investigación de la Escuela de gestión de Transporte.

El 38\% de los encuestados se encuentran entre la edad de 19 a 25 años, mientras que el $17 \%$ están entre la edad de 26 a 32 años. (Salas C. , 2018)

\section{Antecedentes Históricos}

\section{Ministerio de Transporte y Obras públicas del Ecuador}

El Ministerio de Transporte y obras públicas nace mediante el registro oficial del 8 de febrero del 2007, respondiendo a la necesidad de una política integral en el transporte, donde posibilite la planificación, el establecimiento de estrategias y una debida coordinación multimodal e intermodal para que el Ecuador se haga saber que se encuentra en los circuitos globales del transporte.

En consecuencia, se crean subsecretarias las cuales son:

- $\quad$ Subsecretaria de Transporte Vial y Ferroviario

- $\quad$ Subsecretaria de Puertos y Transporte Marítimo y Fluvial

- $\quad$ Subsecretaria de Aeropuertos y Transporte Aéreo

- $\quad$ Subsecretaria de Obras Públicas y Comunicaciones 


\section{Subsecretaria de Transporte vial y Ferroviario}

Esta subsecretaria se responsabilizara de la vialidad terrestre y de ferrocarriles, manejará mediante la Ley Orgánica de Transporte Terrestre, Tránsito y Seguridad Vial, donde, mediante el artículo 5 de la ley anterior mencionada, faculta a la Agencia Nacional de Regulación y control de Transporte terrestre, tránsito y seguridad vial o conocida como Agencia Nacional de Tránsito (ANT, 2014), “controlara y exigirá la capacitación integral, permanente, la formación y tecnificación a conductoras y conductores profesionales y no profesionales y el estricto cumplimiento del aseguramiento social".

Control del transporte terrestre, tránsito y seguridad vial del Ecuador

\section{Dirección de Gestión de Movilidad, Tránsito y Seguridad vial}

Nace de un proceso agregado de valor el cual se evidencia en el anexo 5.

Tabla 2. Servicios ofrecidos por la Dirección de Gestión de Movilidad, Tránsito y Transporte del cantón Riobamba

\section{SERVICIOS DESCRIPCIÓN}

Matriculación Y Realiza la actividad de registrar a todo vehículo que circula mediante Revisión los procesos de matriculación y la revisión técnica vehicular.

El proceso se realiza en conjunto con el sistema de matriculación de la Agencia Nacional de Tránsito y el sistema del Servicio de Rentas Internas. Además la Agencia Nacional de Tránsito gestiona el transporte comercial, escolar y de carga liviana de Riobamba.

Señalización Realiza la actividad de la señalización horizontal y vertical para que los peatones, conductores, ciclistas, motociclistas y otros más, para que en el momento de circular por las vías y veredas de Riobamba lo hagan con toda seguridad.

Seguridad Vial Brinda capacitaciones a los peatones, conductores ciclistas sobre la seguridad en la vía pública, para que su desplazamiento sea de una forma segura

Terminal Registra a todos los vehículos que circulan, lo realiza en conjunto con la Terrestre Agencia Nacional de Tránsito

Fuente: Dirección de Gestión de Movilidad, Tránsito y Transporte de Riobamba. Grupo de Investigación 


\section{Fundamentación Teórica}

\section{Servicio}

Un Servicio representa un conjunto de acciones las cuales son realizadas para servir a alguien, algo o alguna causa. Los servicios son funciones ejercidas por las personas hacia otras personas con la finalidad de que estas cumplan con la satisfacción de recibirlos. La etimología de la palabra nos indica que proviene del latín "Serviťum" haciendo referencia a la acción ejercida por el verbo "Servir ". Los servicios prestados es una comunidad cualquiera están determinados en clases, a su vez estas clases están establecidas de acuerdo a la figura, personal o institucional que lo ofrece o imparte. Existen servicios públicos y servicios especializados. (PAZ, 2012)

\section{Administración}

Para (Ortiz, 2009). La administración es "Proceso integral para planear, organizar e integrara una actividad o relación de trabajo, la que se fundamenta en la utilización de recursos para alcanzar un fin determinado".

La administración también puede definirse como la planificación, la dirección, la organización y el control de las actividades dentro de una organización para la cual se requerirá, talento humano, recursos económicos, tecnológicos para alcanzar las metas y poder mantenerse en el mercado competitivo.

La administración se aplica para todos los niveles de organización como compañías, cooperativas tales como públicas y privadas con el fin de sacar un mejor rendimiento en términos de eficiencia y eficacia.

\section{Calidad de Servicio}

La calidad para (Deming, 1989), es "traducir las necesidades futuras de los usuarios en características medibles, solo así un producto puede ser diseñado y fabricado para dar satisfacción a un precio que el cliente pagara"

La calidad puede ser perciba según (Druker, 1990), estableciendo 5 factores en los cuales se puede evaluar el desempeño de una organización de acuerdo a la satisfacción de sus clientes, estos son:

Para (Cronin \& Taylor, 1992), realizan una investigación sobre el modelo SERVQUAL del libro "Calidad Total en la Gestión de Servicios" (Valarie, Parasuraman, \& Berry, 1993), en donde los investigadores crean un modelo llamado SERVPERF, en donde su modelo manifiesta que solo se enfocaran en las percepciones, ya que el modelo SERQUAL investiga las expectativas y las percepciones. 


\section{Ventajas del modelo SERVPERF}

- Necesita menos tiempo para la realización del cuestionario

- La interpretación y el análisis resultara más fácil de realizarlo

Factores de calidad de servicio por Parasuraman, Valarie y Berry

Tabla3. Factores de calidad

Factores

Fiabilidad

Seguridad

Capacidad de respuesta

Empatía

Intangibilidad

Interacción

humana

\section{Descripción}

Es la forma en la empresa realiza su trabajo desde el primer momento dando, confiabilidad, seguridad y de una manera cuidadosa

La organización tiene que demostrar que se interesa en su cliente para que obtenga una mayor satisfacción estableciendo su confiabilidad, integridad y honestidad.

Fuente: Calidad Total en la Gestión de Servicios (Valarie, Parasuraman, \& Berry, 1993). Según (Eiglier y Langeard, 1989), desde su concepto de servicio explica 3 dimensiones percibidas y adquiridas por el cliente:

\section{Marco Metodológico}

\section{Modalidad de la Investigación}

\section{Cuantitativa}

En la investigación se realizara dicho tipo de diseño ya que se tomara información que se puede contabilizar y consecuentemente tener una base de datos para analizar el mismo que se utilizara para los fines de la investigación (Rodriguez \& Valldeoriola, 2009).

Dentro de esta modalidad podemos encontrar la siguiente investigación:

\section{No Experimental}

Dentro de la modalidad de investigación se va a utilizar el diseño no experimental porque solo se emplea observación y que realizara sin manipular deliberadamente variables, es decir, observar fenómenos tal y como se dan en su contexto natural, para después analizarlos y que 
el investigador no provocara ninguna situación del lugar de estudio (Rodriguez \& Valldeoriola, 2009).

\section{Cualitativo}

Se tomara algunas características del lugar de investigación y los procedimientos que se realizan dentro del lugar de investigación por lo que el investigador realizara la toma la información (Rodriguez \& Valldeoriola, 2009).

\section{Tipos de Investigación}

\section{Investigación Exploratoria}

Nos ofrece obtener un acercamiento al problema para poder estudiarlo, es decir, familiarizarnos con lo que estamos investigando, con esto obtenemos información de carácter inicial para poder seguir con una investigación más profunda.

\section{Investigación Descriptiva}

Utilizaremos de este tipo para poder interpretar, analizar los resultados obtenidos con los instrumentos que utilicemos en la investigación, y plasmarlos para el entendimiento de todos los aspectos que se trate.

\section{Investigación Explicativa}

Este tipo de investigación busca donde se origina los problemas y dar conocimiento de todas las partes que la componen, dando, conclusiones, explicaciones y así enriquecer de conocimiento del presente trabajo de titulación

\section{Investigación de campo}

Es un procedimiento que se lleva a cabo en el ambiente natural de las personas u objetos sobre los que se realiza el estudio, es decir, porque el investigador va a estar muchas veces en el lugar de los hechos para observar el comportamiento del lugar de trabajo y reacciones ante las diferentes situaciones reales.

\section{Investigación bibliográfica}

El investigador tendrá basada su investigación en documentos, repositorios, páginas Web para poder ampliar las bases de la investigación y lo hará referencia a su respectiva norma (APA, 2016).

\section{Investigación Documental}

El investigador concretara la recopilación de información en diversas fuentes de revistas, videos para poder recuperar, analizar, criticar e interpretar los datos obtenidos y registrados 
por anteriores investigadores que han realizado la investigación respecto a las variables a considerar en esta investigación.

\section{Resultados.}

Para este análisis se tomó como quejas las cantidades en donde encontramos mayor insatisfacción y descontento en el usuario.

Los altos porcentajes a ser analizados en forma individual y grado de insatisfacción son los siguientes:

1.- Ruta de transporte público que circule a menos de cinco cuadras de su domicilio $74 \%$

2.- Información de rutas

$60 \%$

3.- Esperan más de 7 minutos

$58 \%$

4.- Necesitan tiempo de viaje más rápido

$57 \%$

5.- Seguridad para atender cualquier eventualidad $\quad 56 \%$

Tabla 4. Tabulación de quejas de los usuarios

\begin{tabular}{lccc}
\hline \multicolumn{1}{c}{ PUNTO ANALIZADO } & FRECUENCIA & PORCENTAJE & $\begin{array}{l}\text { PORCENTAJE } \\
\text { ACUMULADO }\end{array}$ \\
\hline $\begin{array}{l}\text { Ruta de transporte público que circule a } \\
\text { menos de cinco cuadras de su domicilio } \\
74 \%\end{array}$ & 295 & $24 \%$ & 24 \\
$\begin{array}{l}\text { Información de rutas } \\
\text { Esperan más de 7 minutos }\end{array}$ & 238 & $20 \%$ & 44 \\
$\begin{array}{l}\text { Necesitan tiempo de viaje más rápido } \\
\text { Seguridad para atender cualquier } \\
\text { eventualidad }\end{array}$ & 227 & $19 \%$ & 63 \\
TOTAL & 222 & $19 \%$ & 82 \\
\hline
\end{tabular}

Fuente: Análisis matemático en la calidad en el servicio urbano en la ciudad de Riobamba para futuros proyectos de inversión publica 
Tabla 5. Acciones de mejoras A

Descripción de la insatisfacción

\section{ÁREA DE MEJORA N ${ }^{\circ} 1$}

Causas que provocan la insatisfacción

Objetivo a conseguir

Acciones de mejora

Acciones de mejora

Beneficios esperados

\section{ÁREA DE MEJORA N ${ }^{\circ} 2$}

Causas que provocan la insatisfacción

Objetivo a conseguir

Acciones de mejora

Acciones de mejora

Acciones de mejora

Beneficios esperados

\section{ÁREA DE MEJORA N ${ }^{\circ} 3$}

Causas que provocan la insatisfacción

Objetivo a conseguir

Acciones de mejora

Acciones de mejora

Beneficios esperados

\section{Acciones correctivas}

Ruta de transporte público que circule a menos de cinco cuadras de su domicilio $74 \%$

Falta de planificación, actualización

Satisfacción del cliente

1.1.-Reestructuracion de Rutas

1.2.-Reestructuración de Frecuencias

Planificación de la movilidad con visión futura

Información de rutas (trayecto de recorrido)

Desconocimiento de la ciudadanía

Correcto uso por parte del usuario de las rutas determinadas

2.1.-Implementar las Parada definidas para el usuario, establecer señalética informativa en cada parada.

2.2.-Socializacion a través de los medios de comunicación.

2.3.-Programas de educación con escuelas y colegios.

Ciudadanía totalmente informada.

Esperan más de 7 minutos

Pocas unidades de circulación en determinadas frecuencias.

Mejorar el tiempo de espera.

3.1.-Control de las operadoras para su mejor fluidez.

3.2.-Flexibilidad en la normativa y reglamento para aprobar el incremento de unidades según el caso.

Movilidad oportuna y a tiempo

\section{Fuente: Ciudadanía}

Tabla 6. Acciones de Mejoras B

\begin{tabular}{llllll}
\hline \multicolumn{1}{c}{ N } & \multicolumn{1}{c}{ Acciones de mejora } & \multicolumn{1}{c}{ Dificultad } & Plazo & $\begin{array}{l}\text { Impacto } \\
\text { P.-positivo } \\
\text { N.-negativo }\end{array}$ & Priorización \\
\hline 1.1 & Reestructuración de Rutas & Estudio previo & 3 meses & P.-Mejora de Servicio & Urgente \\
1.2 & Reestructuración de Frecuencias & Estudios actualizados & 2 años & $\begin{array}{l}\text { P.--Incremento de } \\
\text { movilidad publica }\end{array}$ & la Urgente \\
& $\begin{array}{l}\text { Implementar paradas definidas } \\
\text { para el usuario, Establecer } \\
\text { señalética informativa en cada } \\
\text { parada. }\end{array}$ & $\begin{array}{l}\text { No hay infraestructura } \\
\text { de paradas definidas }\end{array}$ & 3 meses & $\begin{array}{l}\text { P cambio de cultura al } \\
\text { educar al usuario y al } \\
\text { transportista a respetar las } \\
\text { paradas definidas }\end{array}$ & Urgente
\end{tabular}


2.2 Socialización a través de los medios de comunicación

2.3 Programas de educación con escuelas y colegios

3.1 Control de las operadoras para el incremento de las unidades

Flexibilidad en la normativa y

3.2 reglamento para aprobar el incremento de unidades según el caso.
P. conocimiento

\begin{tabular}{|c|c|c|c|}
\hline Ninguna & 3 meses & $\begin{array}{l}\text { N. no llega al } 100 \% \text { de la } \\
\text { población }\end{array}$ & Urgente \\
\hline $\begin{array}{l}\text { Recurso Humano } \\
\text { adecuado }\end{array}$ & 1 año & $\begin{array}{l}\text { P. marketing boca a boca } \\
\text { en el núcleo familiar }\end{array}$ & Urgente \\
\hline Organización & 1 año & $\begin{array}{l}\text { P. incremento de } \\
\text { Movilidad. }\end{array}$ & Urgente \\
\hline Conflictos de intereses. & & N. Contaminación & \\
\hline $\begin{array}{l}\text { Agencia nacional de } \\
\text { tránsito. }\end{array}$ & 1 año & P. Flexibilidad & Urgente \\
\hline
\end{tabular}

Fuente: Ciudadanía

\section{CONCLUSIONES}

La calidad en el servicio de transporte urbano en la ciudad de Riobamba no satisface las necesidades de los usuarios se encontraron problemas como

$>$ No existen una planificación organizada en la ciudad, lo se me pudo medir en el grado de insatisfacción de los usuarios, tiempo de espera en algunas líneas exceden los 10 minutos,

$>$. No existen información de rutas, peor aún paradas definidas

\section{RECOMENDACIONES:}

- Los organismos correspondientes a nivel nacional deben observar y hacer cumplir las normas y estándares internacionales y nacionales relacionados al transporte terrestre urbano.

- Para generar calidad del servicio se deberá contar con rutas, paradas y señalética debidamente socializada con la ciudadanía para su adecuado uso.

- Realizar capacitación constante a los involucrados y responsables en la calidad del servicio de transporte urbano. 


\section{BIBLIOGRAFIA}

2010, I. C. (2010). http://app.sni.gob.ec/sni-

link/sni/Portal\%20SNI\%202014/FICHAS\%20F/0601_RIOBAMBA_CHIMBORAZO.pdf.

(INEC) Recuperado el SABADO de DICIEMBRE de 2017, de http://app.sni.gob.ec/snilink/sni/Portal\%20SNI\%202014/FICHAS\%20F/0601_RIOBAMBA_CHIMBORAZO.pdf.

2010, s. p. (sabdo de diciembre de 2010). http://app.sni.gob.ec/snilink/sni/Portal\%20SNI\%202014/FICHAS\%20F/0601_RIOBAMBA_CHIMBORAZO .pdf. (Senso poblacional y vivienda 2100) Recuperado el sabado de Diciembre de 2017, de http://app.sni.gob.ec/snilink/sni/Portal\%20SNI\%202014/FICHAS\%20F/0601_RIOBAMBA_CHIMBORA ZO.pdf.

ANT. (2014). Ley Organica de Transporte terrestre Transito y Seguridad Vial.

BanEcuador. (mayo de 2017). https://www.banecuador.fin.ec/noticiasbanecuador/boletines-de-prensa/la-banca-publica-conversa-los-industrialesriobamba/. Recuperado el saBADO de diciembre de 2017

Bangara S, A. (2011). Cobranza ,credito administracon. cobranza, credito administrativo, 1 .

Carrasco, J. (2008). Gestión de procesos. Santiago de Chile: Evuluacion S.A.

COAC, R. (SABADO de DICIEMBRE de 2017). https://cooprio.fin.ec/info/. Recuperado el SABADO de DICIEMBRE de 2017, de https://cooprio.fin.ec/info/.

COMERCIO, E. (LUINES de ABRIL de 2014). http://www.elcomercio.com/actualidad/ecuador/92-empresas-son-generadorasde.html. (EL COMERCIO) Recuperado el SABADO de DICIEMBRE de 2017, de http://www.elcomercio.com/actualidad/ecuador/92-empresas-son-generadorasde.html.

Cronin, J., \& Taylor, S. (1992). Measuring Service Quality: A Reexamination and Extension. American Marketing Association, 55-68.

ECUADOR, B. C. (SABADO de DICIEMBRE de 2017). https://www.bce.fin.ec/documents/pdf/proyecto_bid_bce/Coacsaprobadasxregionact .pdf. (COOP DE AHORRO Y CREDITO) Recuperado el SABADO de DICIEMBRE de 2017, de 
https://www.bce.fin.ec/documents/pdf/proyecto_bid_bce/Coacsaprobadasxregionact .pdf.

Galván, A. C. (2012). EMPRENDIMIENTO PARA LA GESTION DE EMPRESAS CON RESPONSABILIDAD SOCIAL. En A. C. Galván, EMPRENDIMIENTO PARA LA GESTION DE EMPRESAS CON RESPONSABILIDAD SOCIAL. BOGOTA. Recuperado el LUNES de DICIEMBRE de 2017

German Garcia, j. i. (2011). gerencia integral para el siglo XXI. (r. bibliograficas, Ed.) Bogota: Universidad AEN.

GOMEZ, R. C. (2015). ADMINISTRACION DE LA CALIDAD TOTAL. MAR DE PLATA: UNIVERSIDAD MAR DE PLATA.

GUILLO, J. J. (2016). CALIDAD TOTAL FUENTE DE LA VENTAJA COMPETITIVA. En J. J. GUILLO, CALIDAD TOTAL FUENTE DE LA VENTAJA COMPETITIVA. MURCIA: PUBLICACIONES DE LA UNIVERSIDAD DE ALICANTE.

Inversione, D. d. (2012). Dirección de Inteligencia Comercial e Inversione "'"Pro Ecuador. marx, k. (s.f.). https://definicion.deleconomia/. Recuperado el miercoles de diciembre de 2017, de definiciones economicas.

OCTUBRE, C. 4. (SABADO de DICIEMBRE de 2017). http://4deoctubre.net/productos/ahorros.html. (CUATRO DE OCTUBRE) Recuperado el SABADO de DICIEMBRE de 2017, de http://4deoctubre.net/productos/ahorros.html.

PAZ, R. (2012). SERVICIO AL CLIENTE ADMINISTRACION Y CALIDAD. VIGO.

PENNANCE, a. s. (s.f.). DICCIONARIO DE ECONOMIA. BARCELONA: VILASSAR DEL MAR.

Portugal, C. (s.f.). Tesis de Celia portugal. Recuperado el 22 de febrero de 2016, de UNIVERSIDAD TECNICA ESTATAL DE QUEVEDO-TESIS DE CELIA PORTUGAL: https://www.monografias.com/trabajos75\%20analisis-fodaherramienas-planeacion-estrategica

r. (s.f.).

Raquel Ayestaran, C. R. (2012). Planificacion Estrategica y gestion de la Publicidad. Madrid: ESIC EDITORIAL.

Rodriguez, D., \& Valldeoriola, J. (2009). Metodologia de Investigación. Barcelona: Universitat Oberta de Catalunya. 
Salas, C. (2018). Analisis matematico en la calidad en el transporte urbano de la ciudad de Riobamba para futuros proyectos de inversion publica. 2018.

salas, c. (2018). analisis matematico en la calidad en el transporte urbanode riobamba para futuros proyectos de inversion publica. latindex, 20.

Salas, M. (2016). En Salas, \& M, Desarrollodel marketing mi de la empresa de confecciones Lilis (pág. 10). Guayaquil.

Salas, M. (2016). Desarrollo del marketing Mix para incrementar el volumen de ventas de la empresa de confecciones lilis. Guayaquil, Guayas, Ecuador.

Tejada, A. (2003). Los modelos acutales de gestión en las organizaciones, gestión del talento, gestión del conocimiento y gestión por competencias. Psicologia desde el caribe, 115-133.

Valarie, Parasuraman, \& Berry. (1993). Calidad total en la gestión de servicios. Madrid: Diaz de Santos.

Vicuña Ancín, J. M. (2015). El plan estratégico en la práctica. Esic Editorial. Espana: Esic. Editorial.

vistazo, r. (12 de febrero de 2016). El sector textil en cifras. Obtenido de vistazo: http://vistazo.com/seccion/pais/el-sector-textil-en-cifras

WIKIPEDIA. (2017). https://es.wikipedia.org/wiki/Poblaci\%C3\%B3n_activa. (WIKIPEDIA) Recuperado el LUNES de DICIEMBRE de 2017, de https://es.wikipedia.org/wiki/Poblaci\%C3\%B3n_activa.

yachay. (2011). http://www.yachay.gob.ec/asociaciones-de-la-economia-popular-ysolidaria-se-capacitan/. Recuperado el domingo de diciembre de 2107

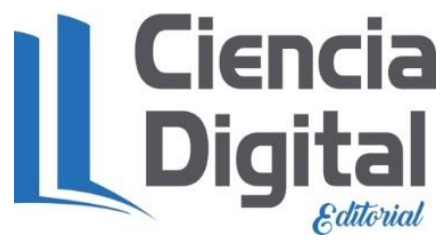




\section{PARA CITAR EL ARTÍCULO INDEXADO.}

Padilla Muñoz, M., Llamuca Llamuca, J., Calderón Limaico, P., \& Villamarín Padilla, J. (2019). Modelos matemáticos y la calidad del servicio aplicados al transporte urbano en Riobamba. Ciencia Digital, 3(2.2), 146-160.

https://doi.org/10.33262/cienciadigital.v3i2.2.466

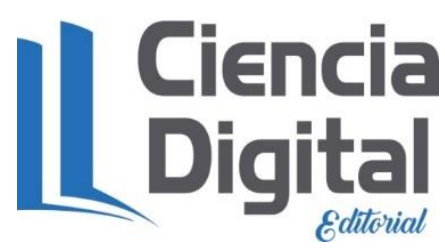

El artículo que se publica es de exclusiva responsabilidad de los autores y no necesariamente reflejan el pensamiento de la Revista Ciencia Digital.

El artículo queda en propiedad de la revista y, por tanto, su publicación parcial y/o total en otro medio tiene que ser autorizado por el director de la Revista Ciencia Digital.
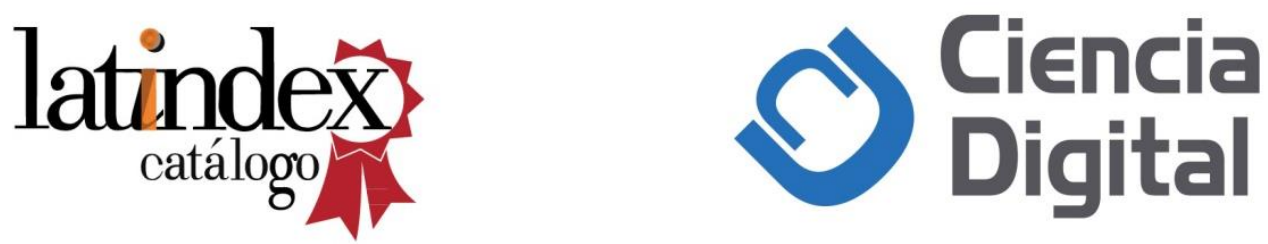